\title{
AN INTERESTING CASE OF DIFFERENTIATED NEUROBLASTOMA- GANGLIONEUROMA OF THE NECK IN A 5 YEAR OLD FEMALE CHILD.
}

\author{
M. Ramani ${ }^{1}$, O. H. Radhika Krishna ${ }^{2}$, K. Ramesh Reddy ${ }^{3}$, Chanakya ${ }^{4}$, R. Sowjanya ${ }^{5}$.
}

\author{
1. Professor, Department of Pathology, Niloufer hospital. \\ 2. Assistant professor, Department of Pathology, Niloufer hospital \\ 3. Professor and Head of the Department, Department of Paediatric Surgery, Niloufer Hospital. \\ 4. $3^{\text {rd }}$ year under graduate, Department of Pathology, Osmania Medical College. \\ 5. Post graduate, Department of pathology, Niloufer hospital.
}

\section{CORRESPONDING AUTHOR:}

Dr. M. Ramani,

Niloufer Hospital for women and child Health,

Red hills Hyderabad, Andhra Pradesh.

E-mail: drmramani@sify.com

\section{HOW TO CITE THIS ARTICLE:}

M. Ramani, O. H. Radhika Krishna, K. Ramesh Reddy, Chanakya, R. Sowjanya. "An Interesting Case of differentiated Neuroblastoma-Ganglioneuroma of the Neck in a 5 year old Female Child". Journal of Evolution of Medical and Dental Sciences 2013; Vol2, Issue 24, June 17; Page: 4298-4301.

ABSTRACT: Ganglioneuroma is a rare, benign, neuroblastic tumor arising mainly from the central or peripheral autonomic nervous system, especially the sympathetic system. The most affected anatomical sites are the posterior mediastinum, retroperitoneum, adrenal gland and head and neck (1). Tumour arises usually from the sympathetic trunk or rarely from the ganglion nodosum of vagus nerve. Here we report a case of 5 year old female child who presented with swelling over the left side of neck. The mass was excised and histopathologically and immunohistochemically diagnosed as ganglioneuroma.

KEYWORDS: Ganglioneuroma, neck, 5 year female child.

INTRODUCTION: Ganglioneuromas are benign tumors that originate from primordial neural crest cells which migrate from the mantle layer of the developing spinal cord to the sympathetic ganglia, adrenal medulla, and other sites. These tumors are well circumscribed and consist of ganglion cells and Schwann cells with few neuroblasts. Ganglioneuromas occur most commonly in children over 10 years of age (2). Peripheral neuroblastic tumors, including neuroblastomas, ganglioneuroblastomas and ganglioneuromas, are rare and constitute only 6\% of tumors in children. Ganglioneuromas are the most differentiated and benign form of Peripheral neuroblastic tumors and the majority of Ganglioneuromas display as slow growing, solitary lesions that may or may not have an effect on neighboring structures (3).

CASE REPORT: A 5 year old female child presented with a swelling over the neck on left side. The swelling was present since 3 yrs. There was on and off fever associated with cough since 3 yrs. Family history was nil remarkable. Palpation revealed a swelling below angle of mandible measuring $5 \mathrm{~cm} \times 4 \mathrm{~cm}$, soft, immobile, non tender. Ultrasonography of neck showed well defined 
heterogenous mass lesion. CT images identified single well circumscribed mass on the left side of the neck.

Provisional diagnosis was either neurofibroma or schwannoma.

GROSS- We received a globular soft tissue mass measuring $5 \mathrm{~cm} \times 4.5 \mathrm{~cm} \times 3 \mathrm{~cm}$. Cut section was solid, grey white and mucoid.

MICROSCOPY- Multiple sections studied from the mass showed large mature ganglion cells, schwann cells and tangled masses of nerve bundles in background of schwannian stroma, suggestive of ganglioneuroma. Immunohistochemistry was positive for Neuron specific enolase.

DISCUSSION: A Ganglioneuroma occurring in the neck was first described by De Quervain" in 1889. Dunn 15 reported four cases in the neck in 1915, and in 1935 McFarland and Sappington 16 reported 18 cases, also of cervical origin (4).

Ganglioneuroma is a rare benign, neurogenic tumour arising from the sympathetic nervous system. It belongs to the family of neuroblastic tumours which includes neuroblastoma and ganglioneuroblastoma. Generally the tumour is unique and unilateral, whereas multiple localizations associated with neurofibromatosis have been reported. These neoplasms arise from neural crest neuroblasts that under normal conditions migrate into the adrenal medulla and sympathetic ganglia during fetal development. Solitary Ganglioneuromas most commonly occur in infants and young children, slightly more often in girls than boys, with a female-male ratio of about $3: 1$. The majority are diagnosed before the patient is 10 years old.

Ganglioneuromas are typically located in the thoracic cavity (60-80\%, posterior mediastinum), the abdominal cavity (10-15\%, adrenal gland, retroperitoneum, pelvic, sacral and coccygeal sympathetic ganglia, and the organ of Zuckerkandl) and the cervical region (5\%). Other less common sites are the middle ear, the parapharynx, the skin, the orbital space and the gastrointestinal tract (5)

Ganglioneuromas are usually asymptomatic regardless of their size. If the tumor is in the mediastinum, it may cause chest pain, cough, and difficulty in breathing. If the tumor is in the retroperitoneal space, it may result in abdominal pain and distension. These tumors may be hormonally active and hypertension, diarrhea, flushing and virilization may occur as a result the secretion of catecholamine, vasoactive intestinal polypeptide, or androgenic hormone.

Imageology is helpful for preoperative diagnosis. On FNAC, it is difficult to differentiate from other neurogenic neoplasms such as schwannomas and neurofibromas, and an incorrect diagnosis may be made when FNAB (fine needle aspiration biopsy) fails to yield the ganglion cells (5). A specific diagnosis of ganglioneuroma is confirmed only by pathological study. The pathologic features of Ganglioneuromas are a blend of mature ganglion cells randomly distributed along with a component of spindle cells that corresponds to schwann cells and endoneurial cells. These components can be embedded in a fibromyxoid or fibrillar matrix.

The differential diagnosis includes paraganglioma, neurilemmoma and neurofibroma. Ganglioneuroblastomas, the most important differential diagnosis for tumors found in these areas, affects mainly the pediatric age group and are most commonly diagnosed within the first 4 years of life (1). Ganglioneuromas have no Immature elements, atypia, mitotic figures, intermediate cells or necrosis. The presence of any these tissue characteristics excludes the diagnosis of ganglioneuroma $(6)$. 
IMMUNOHISTOCHEMISTRY: Positive staining is observed with S-100, synaptophysin, chromogranin A, NSE. Neurogenic tumours are insensitive to the radiotherapy and chemotherapy, so the main treatment for the ganglioneuroma is surgical excision. When complete resection is performed for the well encapsulated tumours, the prognosis is good. For the unencapsulated tumours, preoperative operation should be careful. In the tumour containing immature content, regular follow should be taken to prevent recurrence (1)

Rarely Ganglioneuroma may become malignant and metastasize or recur. In our case, operative findings showed well encapsulated tumour arising from left side of neck, complete excision was possible. Surgical excision is the treatment of choice in these cases, both to confirm the diagnosis and to prevent any further tumour growth and consequent compression of the adjacent structures (7)

CONCLUSION: Ganglioneuroma of the cervical region is an uncommon benign soft tissue neoplasm of neuroblastic origin. Although very uncommon, Ganglioneuromas should be considered in the differential diagnosis of pediatric soft tissue tumors of the head and neck. It should be differentiated from other soft tissue tumors because of difference in the treatment and prognosis. It should be considered in the evaluation of newborns or young infants presenting with solitary or multiple tumors. It is a benign, slow growing tumor and since it is difficult to distinguish from other tumors due to lack of image findings diagnosis is always made histologically ( 8 )

\section{REFERENCES:}

1. Zhang Zebing, Shang Jianwei, Chen Yan, Gao Yan; clinico pathological characteristics of neck ganglioneuroma; oral med pathol 2008, vol.12, 131-134.

2. Celeste Gary, Hugh Robertson, Bernardo Ruiz, Vladimir Zuzukin, Rohan R. Walvekar; Retropharyngeal Ganglioneuroma Presenting with Neck Stiffness. Skull Base- September 2010; 20(5): 371-374.

3. Junli Ma, Limin Liang And Hongchen Liu - Multiple Cervical Ganglioneuroma; Oncology Letters 4; 509-512, 2012.

4. Douglas A. Danosos, Vladimir B. Santos, Mauro L. Ruffy, Ira A. Polisar, and Roosevelt Torno ; Ganglioneuroma Of The Parapharyngeal Space ; September 1980 ; 56( 7), 619.

5. Marco A Ponce-Camacho, Ricardo Diaz de Leon-Medina, Ivett Miranda-Maldonado, Raquel Garza-Guajardo, Jorge Hernandez-Salazar and Oralia Barboza-Quintana - A 5 year old girl with a congenital ganglioneuroma diagnosed by fine needle aspiration biopsy, CytoJournal 2008, vol. 5, 5 .

6. O Adam, Es Boia, Rodica Ilie, Ramona Mandrusca -Ganglioneuroma, Jurnalul Pediatrului , July-December 2007, Vol. 10 , 39-40,

7. Hidenori Yokoi, Atsushi Arakawa, Ayako Inoshita and Katsuhisa Ikeda - Novel use of a Weerda laryngoscope for transoral excision of a cervical ganglioneuroma, Journal of Medical Case Reports 2012, vol. 6, 88.

8. Prasad K Shetty, Balaiah K, Gnana Prakash S, Prasanna K Shetty- Ganglioneuroma Always A Histopathological Diagnosis, Online Journal of Health and Allied Sciences, Dec 2010, Vol. 9(4), 19. 


\section{CASE REPORT}
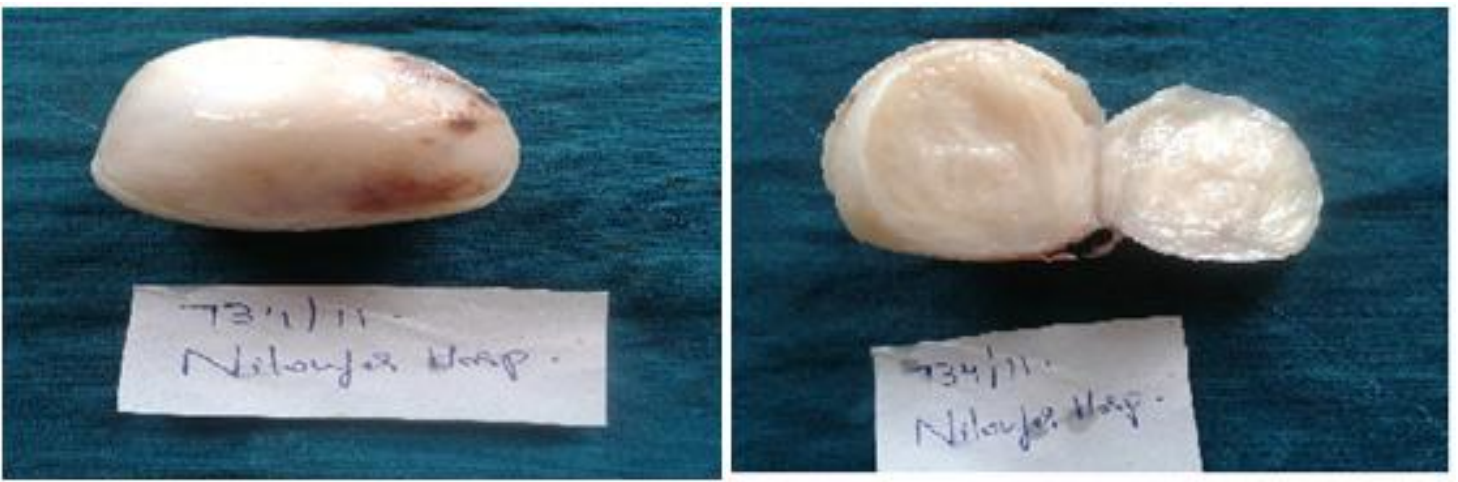

Fig-1: Globular soft tissue mass measuring $5 \mathrm{~cm}$ x $4.5 \mathrm{~cm} \times 3 \mathrm{~cm}$. Cut section was solid, grey white, mucoid.

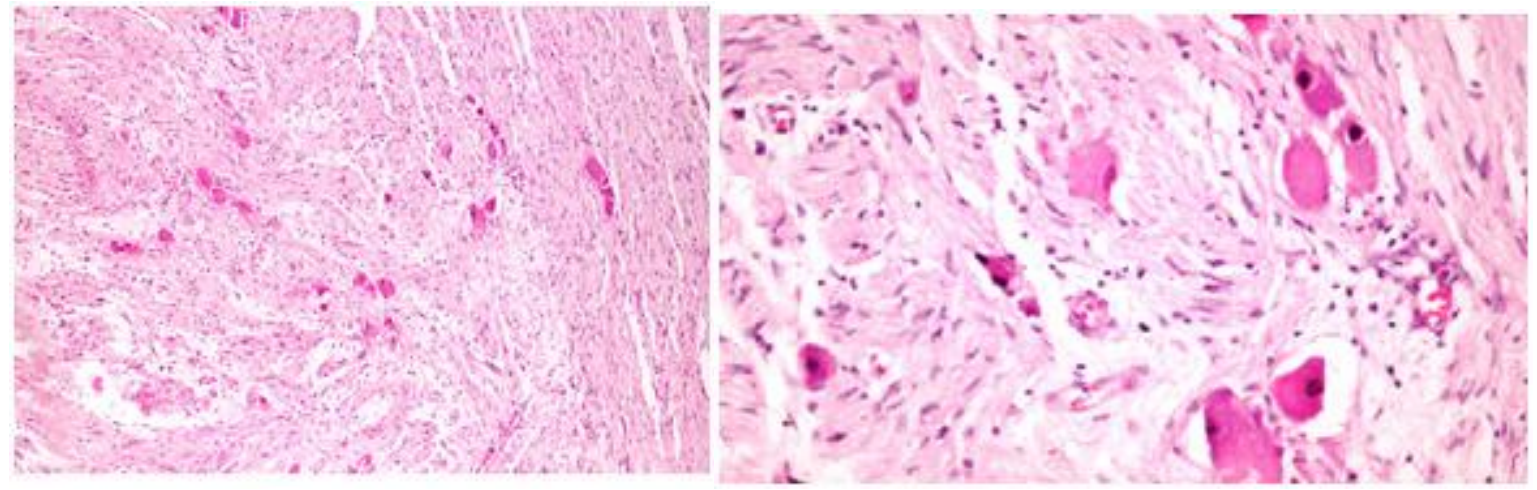

Fig-2: H\&E (10X) and (40X) showing mature ganglion cells and schwannian stroma.
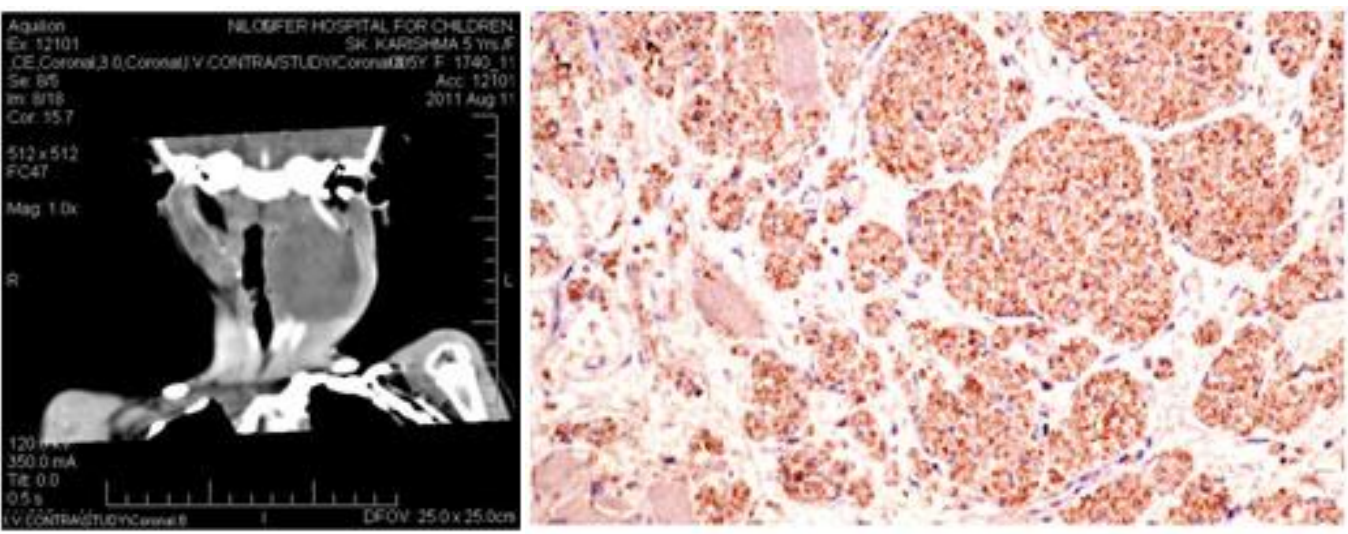

Fig-3: a) well defined mass lesion in the left upper cervical region.

b) IHC positive for Neuron specific enolase. 